Results Transmission contributions from each site have greater uncertainty when more routes may transmit, when all routes may transmit, the oropharynx can contribute $0-100 \%$ of all transmissions. In contrast, when only anal or oral sex may transmit, transmission from the oropharynx can account for only $0-25 \%$ of transmission. Intervention effectiveness against transmission from each site also has greater uncertainty when more routes may transmit.

Conclusion Multiple routes of transmission leads to great uncertainty. Even under ideal conditions (i.e., when site-specific gonococcal prevalence, relative rates of specific sex acts, and duration of infection at each anatomical site are known and do not vary), the relative importance of different anatomical sites for gonococcal transmission cannot be inferred with precision. This result is generalizable to any other infection where multi-site infection leads to multiple routes of transmission. Additional data informing per act transmissibility are needed to understand site-specific gonococcal infection transmission. This understanding is essential for predicting population-specific intervention effectiveness.

Disclosure No significant relationships.

\section{S16.3 ANATOMICAL SITES OF INFECTION: BEHAVIOURAL CONSIDERATIONS FOR STI PREVENTION}

Catherine Mercer*. University College London, Centre for Population Research in Sexual Health and HIV, Institute for Global Health, London, UK

\subsection{6/sextrans-2019-sti.75}

Many industrialised countries have witnessed a broadening of sexual repertoires, including increases in reported heterosexual oral, and in particular, anal sex, and same-sex behaviour in women, while among MSM, oral sex remains more prevalent than anal sex. Condoms, when used correctly, are highly-effective in preventing STI transmission through penetrative sex, yet their use remains suboptimal. For MSM, this may partly reflect the effectiveness of biomedical interventions for HIV such as treatment as prevention and pre-exposure prophylaxis. For heterosexuals, pregnancy prevention often trumps STI concerns, with more reliable and less intrusive contraception used for vaginal sex, while condoms are seldom used for heterosexual anal sex, or oral sex regardless of gender.

Given these behavioural trends, it is unsurprising that a large proportion of STI transmission is thought to occur extra-genitally. Among MSM attending US sexual health clinics, more than half of GC/CT infections were not in the urethra, and most MSM with extra-genital GC/CT infections did not have concurrent urethral infections. Extra-genital infections are more often asymptomatic, a potential reservoir for transmission, and undetected antibiotic resistant strains may spread resistance. STI prevention efforts must therefore include targeting extra-genital infections.

Efforts to change sexual practice, e.g. promoting condom use for oral sex and/or sexual positioning, are unlikely to have significant impacts, but opportunities exist beyond the bedroom. Raising public awareness about the potential for, and consequences of, extra-genital infection may encourage disclosing sexual behaviour to clinicians and appropriate sitespecific testing. Educating clinicians - especially non-specialists - about the importance of asking all patients about their sexual practices and testing for extra-genital STIs accordingly may also be helpful. Such endeavours could result in the greater detection of extra-genital infections but cost-effective strategies need determining. As such, a multifaceted approach including evidence-based behavioural and biomedical interventions is likely to yield the greatest health gains.

Disclosure No significant relationships.

\section{S16.4 DESIGNING AN APPROACH TO DEAL WITH EXTRAGENITAL SEXUALLY TRANSMITTED INFECTIONS: DO WE HAVE THE DATA WE NEED?}

Jeanne Marrazzo*. University of Alabama, Medicine, Birmingham, USA

\subsection{6/sextrans-2019-sti.76}

This symposium entitled 'Anatomical Sites of Infection: Biomedical, Modeling, Behavioral, and Programmatic Considerations for STI Prevention' will focus on the implications that extragenital (defined as infections that occur outside of the cervix and urethra) sexually transmitted infections have for various dimensions of STI care and prevention. These infections are common, particularly among men who have sex with men (rectal and pharyngeal) and heterosexual women (both sites as well). Yet, we have little understanding of the pharmacokinetics of commonly used antimicrobials at these sites, the natural history of the infections at these sites, and as a critical corollary, how much of a role these sites have in providing a meaningful reservoir for sustaining transmission in populations at risk. We need to have a better understanding of these parameters before we can intelligently design screening protocols and intervention studies. This presentation will explore these issues and allow ample time for discussion of these challenges.

Disclosure No significant relationships.

\section{S17 - CLINICAL ISSUES IN WOMEN'S HEALTH AND STI}

Wednesday, July 17, 2019
10:45 AM - 12:15 PM

\section{S17.1 HPV SCREENING - NEW EVIDENCE AND CURRENT STATE OF THE ART}

Suzanne Garland*. The University of Melbourne, Obstetrics and Gynaecology, Parkville, Australia

\subsection{6/sextrans-2019-sti.77}

HPV-vaccination programs constitute major public-health initiatives worldwide and have been introduced into National immunisation programs in over 80 countries, although most are in high income countries. Programs were implemented around 10 years ago: where high coverage of target populations especially with catch-up programs, the impact and effectiveness on HPV infection and disease has been remarkable. For the quadrivalent vaccine $(6 / 11 / 16 / 18)$ there have been reductions of $\sim 90 \%$ for $\mathrm{HPV}$ vaccine type infections, $\sim 90 \%$ for genital warts, $\sim 45 \%$ for low-grade cytological cervical abnormalities, $\sim 60 \%$ for cervical histologically-proven highgrade abnormalities [HSIL], in colposcopic referrals, and 